\title{
Probing the Hydrophobic Effect of Noncovalent Complexes by Mass Spectrometry
}

\author{
Claudia Bich, Samuel Baer, Matthias C. Jecklin, and Renato Zenobi \\ Department of Chemistry and Applied Biosciences, ETH Zurich, Zurich, Switzerland
}

The study of noncovalent interactions by mass spectrometry has become an active field of research in recent years. The role of the different noncovalent intermolecular forces is not yet fully understood since they tend to be modulated upon transfer into the gas phase. The hydrophobic effect, which plays a major role in protein folding, adhesion of lipid bilayers, etc., is absent in the gas phase. Here, noncovalent complexes with different types of interaction forces were investigated by mass spectrometry and compared with the complex present in solution. Creatine kinase (CK), glutathione S-transferase (GST), ribonuclease S (RNase S), and leucine zipper (LZ), which have dissociation constants in the $\mathrm{nM}$ range, were studied by native nanoelectrospray mass spectrometry (nanoESI-MS) and matrix-assisted laser desorption/ ionization mass spectrometry (MALDI-MS) combined with chemical cross-linking (XL). Complexes interacting with hydrogen bonds survived the transfer into gas phase intact and were observed by nanoESI-MS. Complexes that are bound largely by the hydrophobic effect in solution were not detected or only at very low intensity. Complexes with mixed polar and hydrophobic interactions were detected by nanoESI-MS, most likely due to the contribution from polar interactions. All noncovalent complexes could easily be studied by XL MALDI-MS, which demonstrates that the noncovalently bound complexes are conserved, and a real "snap-shot" of the situation in solution can be obtained. (J Am Soc Mass Spectrom 2010, 21, 286-289) (C 2010 American Society for Mass Spectrometry

$\mathrm{E}$ lectrospray is an exceptionally soft ionization technique, which has allowed the observation of numerous noncovalent complexes, including protein-protein, protein-small molecule, and proteinDNA complexes [1, 2]. A major question is still whether the information obtained from gas-phase results is representative of the species present in solution. The term "noncovalent bonding" in biochemistry generally summarizes three types of intermolecular forces: electrostatic interactions (e.g., salt bridges), dipolar interactions (e.g., hydrogen bonds), and van der Waals interactions (e.g., hydrophobic interactions) [3]. In addition, protein-water interactions often play a major role in complex stabilization. In solution, hydrophobic interactions are very weak compared with the hydrogen bonds between water molecules. Hence, it is not the hydrophobic forces that are the actual reason for the interaction of nonpolar binding partners, but rather the surrounding water molecules that "force" hydrophobic binding partners to aggregate. This solvent-driven process is called the hydrophobic effect, and plays a major role in protein folding, adhesion of lipid bilayers, partitioning effects of drugs and metabolites, and many other aggregation phenomena in chemistry and biology [4-6]. The hydrophobic effect is defined by the thermodynamics of mixing hydrophobic compounds with

Address reprint requests to Professor R. Zenobi, Department of Chemistry and Applied Biosciences, ETH Zurich, Honggerberg HC1 E 329, CH-8093 Zurich, Switzerland. E-mail: zenobi@org.chem.ethz.ch water, and is dominated by large, entropic factors [4, 7]. Obviously, in the absence of a hydrate shell, the hydrophobic effect is absent for gaseous proteins [4, 8]. Conversely, electrostatic and polar interactions are stronger in the gas phase compared with solution phase [2].

Considering native protein structures, it has been shown that regions stabilized by hydrophobic interactions in folded cytochrome $c$ unfold upon removal of water [9]. Dehydration of proteins in vacuo has also been studied using computer simulations. The structure of all proteins studied was affected upon removal of the water shell, and the hydrophobic parts of the exposed surface area were larger compared with solution phase (due to the loss of the hydrophobic effect) [10]. Regarding noncovalent interactions, entropy also seems to be the major driving force for dissociation in the gas phase [11]. Nonetheless, there is some evidence that hydrophobic contributions do affect the gas-phase stability of complexes, although this is observed only in combination with electrostatic interactions [12]. Complexes that exist in solution mainly due to hydrophobic effects are likely to dissociate in the gas phase [11,13-16]. When studying noncovalent complexes by ESI-MS, it is known that by increasing the ionic strength or adding organic solvents to the aqueous buffer will affect the hydrophobic effect in solution [17]. Moreover, the intermolecular interaction forces studied by ESI-MS are sensitive to other effects, such as changes in temperature [18], solution $\mathrm{pH}$ [19], and source pressure [20], which can also lead to dissociation of noncovalent complexes.
(C) 2010 American Society for Mass Spectrometry. Published by Elsevier Inc. $1044-0305 / 10 / \$ 32.00$

doi:10.1016/j.jasms.2009.10.012
Published online October 28, 2009

Received August 29, 2009

Revised October 15, 2009

Accepted October 15, 2009 
Matrix-assisted desorption/ionization (MALDI) is another soft ionization method that allows studying noncovalent biomolecular complexes by MS. Although noncovalent intermolecular interactions are usually lost during MALDI sample preparation or during the laser desorption/ionization step, some special techniques permit detection of noncovalent complexes, e.g., first shot experiments [21], particular laser/matrix combinations, and varying the sample preparation conditions [22]. By far the most promising and most general approach is the stabilization of the complex subunits by chemical cross-linking (XL) before MALDI-MS. XL prevents the protein complexes from being disrupted throughout the sample preparation and the desorption/ionization processes [23]. This is clearly an advantage of the XL MALDI-MS approach compared with ESI-MS, since changes in the noncovalent interaction forces during transmission from solution to gas phase are irrelevant.

\section{Experimental}

CK, GST, RNaseS, and sinapinic acid (SA), were obtained from Sigma-Aldrich (Buchs, Switzerland). LZ GCN4 (MW = $4.24 \mathrm{kDa})$ was kindly provided by Y. Nikolaev. Crosslinking was performed using synthesized 1,1'-(suberoyldioxy)bis azabenzotriazole (SBAT) [24].

ESI mass spectra were obtained using a quadrupole time-of-flight mass spectrometer (Q-TOF ULTIMA; Waters/Micromass, Manchester, UK) equipped with an automated nanoESI system (Nanomate 100; Advion Biosciences, Ithaca, NY, USA). The protein samples were desalted before nanoESI-MS analysis using MicroBioSpin columns (MWCO 6000; Bio-Rad, Hercules, CA, USA). To study the protein samples under "native-like" conditions, they were dissolved in $50 \mathrm{mM}$ ammonium acetate buffer at $\mathrm{pH} 7.0$ to give a final concentration of around 5-10 $\mu \mathrm{M}$.

Experiments with the LZ were performed using a commercial MALDI-TOF-MS instrument (Axima CFR; Shimadzu/Kratos Analytical, Manchester, UK). Other experiments were performed using a MALDI-TOF-MS (Reflex IV; Bruker GmbH, Bremen, Germany), equipped with a high-mass detector (CovalX AG; Zürich, Switzerland). For all MALDI-MS experiments, aqueous protein stock solutions were diluted using $10 \mathrm{mM}$ phosphate buffer (PB) at $\mathrm{pH}$ 8.0. $10 \mu \mathrm{L}$ of each protein sample were incubated with $1 \mu \mathrm{L}$ cross-linker solution $(2 \mathrm{mg} / \mathrm{mL}$ SBAT in DMF) for $1 \mathrm{~h}$. One $\mu \mathrm{L}$ of the sample was mixed with 1 $\mu \mathrm{L}$ of freshly prepared SA solution $(10 \mathrm{mg} / \mathrm{mL}$ in water: acetonitrile $(1: 1, \mathrm{vol} / \mathrm{vol})$ containing $0.1 \%$ trifluoric acid). One $\mu \mathrm{L}$ of this mixture containing around 5-10 $\mu \mathrm{M}$ protein was spotted onto the MALDI sample plate.

\section{Results and Discussion}

CK consists of two identical subunits with a $\mathrm{K}_{\mathrm{D}}$ of the homodimer $(\mathrm{MW}=86 \mathrm{kDa})$ of around $50 \mathrm{nM}$ [25]. The crystal structure of the enzyme indicates that at least eight hydrogen bonds at the interface contribute to the dimer cohesion [26]. Theoretically, with a $K_{D}$ of $50 \mathrm{nM}$ and a total protein concentration of $10 \mu \mathrm{M}, 93 \%$ should be in the form of dimer. When studied by nanoESI-MS under native-like conditions, almost full dimerization (89\%) was observed (Figure 1a), which has also been found by other groups [19]. The interaction strength of the two monomer units is thus large enough to survive the ionization and transmission processes. When $C K$ is studied by the XL MALDI-MS approach, also almost full complexation (91\%) can be detected (Figure 1b).

GST exists as stable homodimer with mixed polar and hydrophobic interactions at the subunit interface [27] that contribute to the stabilization of the complex [28]. The $\mathrm{K}_{\mathrm{D}}$ of GST dimerization must be in the low $\mathrm{nM}$ range, since no monomer was observed in solution using different methods [29]. The nanoESI mass spectrum of GST sprayed under native-like conditions showed signals corresponding to both monomer and homodimer (Figure 1c). The survival of the homodimer in the gas phase indicates that the polar interactions keep the structure intact. Again, the control experiment with XL MALDI-MS showed almost exclusively the GST homodimer (Figure 1d).

RNase $S$ consists of two subunits, the S-peptide (S-Pep) and the S-protein (S-Prot). The commercial RNase $S$ sample is a mixture of two S-peptides: residues 1-19 (S-Pep (19), MW = $2095 \mathrm{Da}$ ) and residues 1-20 (S-Pep (20), MW = $2166 \mathrm{Da})$ as well as two S-proteins, S-Prot(20), MW = $11534 \mathrm{Da}$, encompassing residues 20-124, and S-Prot(21), MW = 11447 $\mathrm{Da}$, encompassing residues 21-124. In solution, S-Pep and S-Prot are noncovalently associated to form the active form of RNase $S$. The noncovalent interactions between the two fragments of RNase $S$ in solution are largely based on hydrophobic interactions with a $\mathrm{K}_{\mathrm{D}}$ of around $1 \mathrm{nM}[13,16]$. However, when studied by native nanoESI-MS, only a very small amount of complex was observed (Figure 1e). The relative abundance of RNase $\mathrm{S}$ complex is obviously quite heavily dependent on the ESI interface conditions [16, 30]. The results reported here were obtained using the Nanomate after optimizing the interface conditions. Cytidine nucleotides are also known to bind to RNase $S$ with solution $K_{D} S$ in the $\mu \mathrm{M}$ range. It has been demonstrated that despite the high affinity of S-Pep to S-Prot in solution, in the gas phase this interaction is lost, as opposed to the "weaker" cytidine-S-Prot binding [16]. Therefore, a large contribution of the hydrophobic effect in solution is proposed for the S-Pep $\bullet S$-Prot complex, which is expected to be lost in the gas phase. In contrast, the XL MALDI mass spectra clearly show the S-Pep $\bullet$ S-Prot complex with $88 \%$ relative intensity (Figure 1f). Unfortunately, the $\mathrm{XL}$ reaction is often incomplete due to hydrolysis of one end of the $\mathrm{XL}$ reagent, thus some unbound protein monomer, produced by the MALDI process, can be observed [31].

LZ can form an active dimer with a well-defined conformation. Hydrophobic amino acids (typically leucines) are responsible for stabilizing the dimer in solution [32]. 


\section{nanoESI}
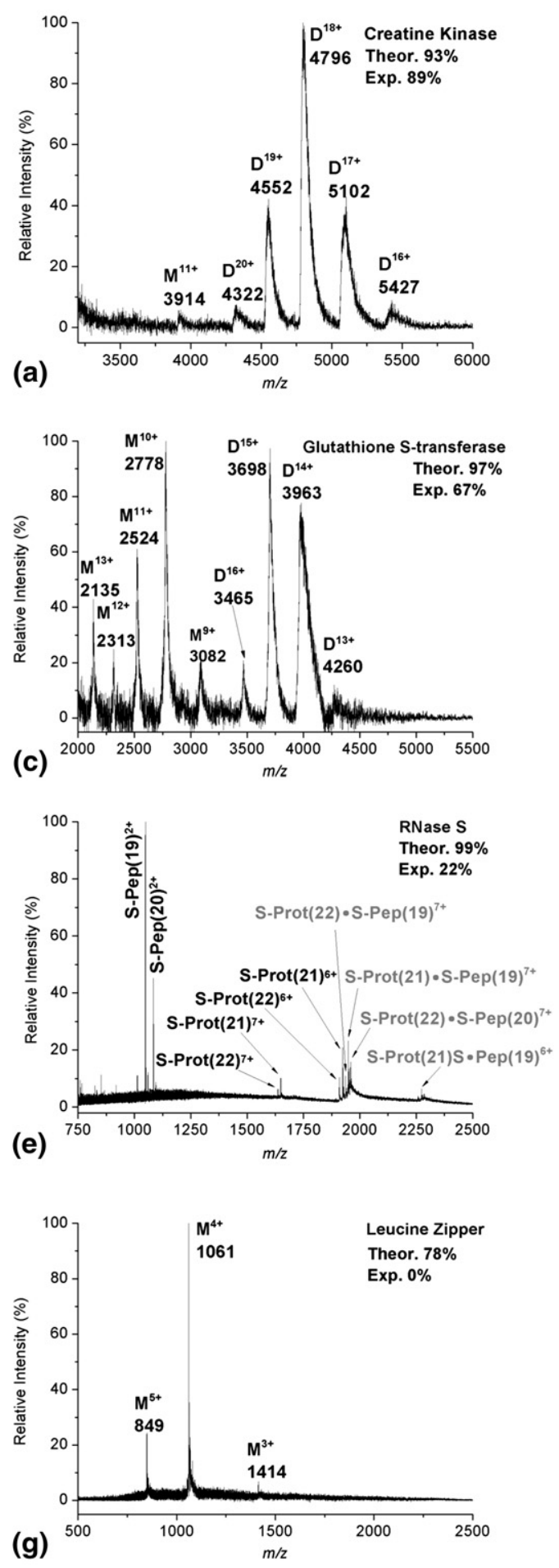

\section{$\mathrm{XL}+\mathrm{MALDI}$}
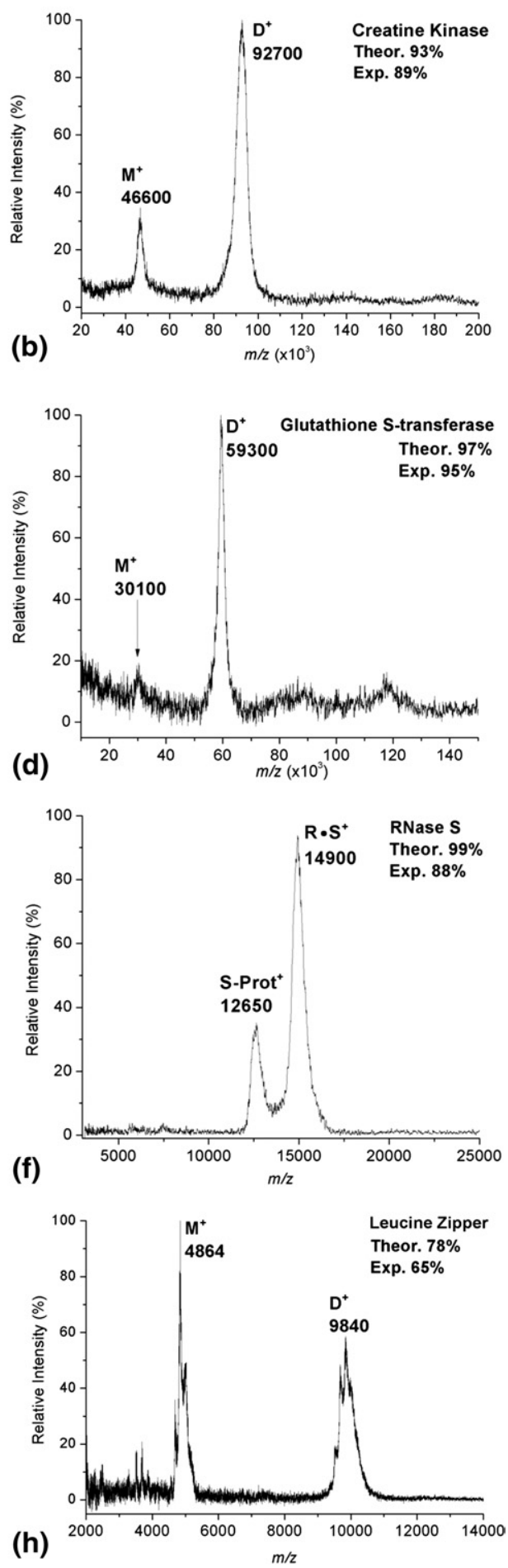

Figure 1. Mass spectra of the protein complexes studied by nanoESI (left) and MALDI (right). The theoretical (calculated from the $\mathrm{K}_{\mathrm{D}}$ values for $10 \mu \mathrm{M}$ total protein concentration) and the experimental percentage of complex are indicated.

The solution $\mathrm{K}_{\mathrm{D}}$ values of the $\mathrm{LZ}$ dimer, which have been determined by various methods, are around 0.5 $\mu \mathrm{M}$ [33]. When studied by nanoESI-MS under nativelike conditions, no signal corresponding to the LZ dimer was observed despite the low $\mathrm{K}_{\mathrm{D}}$ in solution (Figure 1g). Therefore, we conclude that the hydrophobic effect plays the dominant role in stabilizing the LZ dimer in solution. Small amounts $(<25 \%)$ of LZ dimer 
have previously been detected for a modified LZ peptide in combination with a different buffer and instrument [34]. These results are consistent with our findings, since the small amount of complex detected does not reflect the situation in solution, where $78 \%$ of complex should be present. Once more, studying this system with XL MALDI-MS easily allowed detection of $65 \%$ of the LZ dimer (Figure $1 \mathrm{~h}$ ).

In principle, the crosslinked complexes could also have been investigated by ESI-MS for all these systems. However, ESI mass spectra of crosslinked protein complexes are extremely complex because of the multiple species present (varying types of crosslinkers attached, intralinks, dead-end crosslinkers, plus different charges), which are difficult to resolve even in the low $\mathrm{m} / \mathrm{z}$ range. Moreover, due to the excess of XL chemicals needed for such experiments, additional purification would be necessary.

\section{Conclusions}

NanoESI-MS is well suited to analyze noncovalent interactions, and various systems have been successfully investigated thus far [1, 2]. It is well known that electrostatic and polar interactions survive in the gas phase and are even enhanced compared with solution [2]. However, when the interaction in solution is determined by the entropy-driven hydrophobic effect, nanoESI-MS fails to give accurate results representative of the solution equilibria. Similar findings have been reported by other groups [16], including the study of hydrophobic ligands binding to protein targets [13-15]. If mixed polar/hydrophobic interactions are present (GST), the majority of the complex survives the transfer from solution into gas phase, most likely due to the polar fraction [16]. XL MALDI-MS also allows investigation of noncovalent complexes. The amount of dimer detected is in this case independent of the nature of the noncovalent interaction. However, hydrolysis of the reactive group has to be taken into account [24]. These findings demonstrate that in some situations, $\mathrm{XL}$ MALDI-MS may be preferable for studying noncovalent interactions since no bias due to the nature of the interactions and the hydrophobic effect is present.

\section{Acknowledgments}

The authors acknowledge support for this work by the Swiss National Science Foundation, grant no. 200020-124663. The authors thank Y. Nicolaev for sample donation.

\section{References}

1. Loo, J. A. Studying Noncovalent Protein Complexes by Electrospray Ionization Mass Spectrometry. Mass Spectrom. Rev. 1997, 16, 1-23.

2. Daniel, J. M.; Friess, S. D.; Rajagopalan, S.; Wendt, S.; Zenobi, R. Quantitative Determination of Noncovalent Binding Interactions Using Soft Ionization Mass Spectrometry. Int. J. Mass Spectrom. 2002, 216, 1-27.

3. Berg, J.; Tymoczko, J.; Stryer, L. Biochemistry 5th ed., Series Michelle Julet; Freeman: New York, 2002; pp 43-51.

4. Southall, N. T.; Dill, K. A.; Haymet, A. D. J. A View of the Hydrophobic Effect. J. Phys. Chem. B 2002, 106, 521-533.

5. Pace, C. N.; Shirley, B. A.; McNutt, M.; Gajiwala, K. Forces Contributing to the Conformational Stability of Proteins. FASEB J. 1996, 10, 75-83.
6. Ball, P. Water as an Active Constituent in Cell Biology. Chem. Rev. 2008, 108, 74-108.

7. Widom, B.; Bhimalapuram, P.; Koga, K. The Hydrophobic Effect. Phys. Chem. Chem. Phys. 2003, 5, 3085-3093.

8. Kauzmann, W. Some Factors in the Interpretation of Protein Denaturation. Adv. Protein Chem. 1959, 14, 1-63.

9. Breuker, K.; McLafferty, F. W. The Thermal Unfolding of Native Cytochrome $c$ in the Transition from Solution to Gas Phase Probed by Native Electron Capture Dissociation. Angew. Chem. Int. Ed. 2005, 44, 4911-4914.

10. Patriksson, A.; Marklund, E.; van der Spoel, D. Protein Structures Under Electrospray Conditions. Biochemistry 2007, 46, 933-945.

11. Laskin, J.; Futrell, J. H. Entropy is the Major Driving Force for Fragmentation of Proteins and Protein-Ligand Complexes in the Gas Phase. J. Phys. Chem. A 2003, 107, 5836-5839.

12. Franski, R.; Gierczyk, B.; Schroeder, G.; Franska, M.; Wyrwas, B. Do Hydrophobic Interactions Exist in the Gas Phase? Rapid Commun. Mass Spectrom. 2008, 22, 1339-1343.

13. Robinson, C. V.; Chung, E. W.; Kragelund, B. B.; Knudsen, J.; Aplin, R. T.; Poulsen, F. M.; Dobson, C. M. Probing the Nature of Noncovalent Interactions by Mass Spectrometry. A Study of Protein-CoA Ligand Binding and Assembly. J. Am. Chem. Soc. 1996, 118, 8646-8653.

14. Wu, Q.; Gao, J.; Joseph-McCarthy, D.; Sigal, G. B.; Bruce, J. E.; Whitesides, G. M.; Smith, R. D. Carbonic Anhydrase-Inhibitor Binding: From Solution to the Gas Phase. J. Am. Chem. Soc. 1997, 119, 1157-1158.

15. Bovet, C.; Wortmann, A.; Eiler, S.; Granger, F.; Ruff, M.; Gerrits, B.; Moras, D.; Zenobi, R. Estrogen Receptor-Ligand Complexes Measured by Chip-Based Nanoelectrospray Mass Spectrometry: An Approach for the Screening of Endocrine Disruptors. Protein Sci. 2007, 16, 938-946.

16. Yin, S.; Xie, Y.; Loo, J. A. Mass Spectrometry of Protein-Ligand Complexes: Enhanced Gas-Phase Stability of Ribonuclease-Nucleotide Complexes. J. Am. Soc. Mass Spectrom. 2008, 19, 1199-1208.

17. Li, Y.; Heitz, F.; Le Grimellec, C.; Cole, R. B. Hydrophobic Component in Noncovalent Binding of Fusion Peptides to Lipids as Observed by Electrospray Mass Spectrometry. Rapid Commun. Mass Spectrom. 2004, 18, 135-137.

18. Goodlett, D. R.; Ogorzalek Loo, R.; Loo, J. A.; Wahl, J. H.; Udseth, H. R.; Smith, R. D. A Study of the Thermal Denaturation of Ribonuclease S by Electrospray Ionization Mass Spectrometry. J. Am. Soc. Mass Spectrom. 1994, 5, 614-622.

19. Liang, Y.; Du, F.; Sanglier, S.; Zhou, B.; Xia, Y.; Van Dorsselaer, A.; Maechling, C.; Kilhoffer, M.; Haiech, J. J. Unfolding of Rabbit Muscle Creatine Kinase Induced by Acid. J. Biol. Chem. 2003, 278, 30098-30105.

20. Tahallah, N. Pinkse, M. Maier, C. S. Heck, A. J. The Effect of the Source Pressure on the Abundance of Ions of Noncovalent Protein Assemblies in an Electrospray Ionization Orthogonal Time-of-Flight Instrument. Rapid Commun. Mass Spectrom. 2001, 15, 596-601.

21. Strupat, K.; Sagi, D.; Bonisch, H.; Schafer, G.; Peter-Katalinic, J. Oligomerization and Substrate Binding Studies of the Adenylate Kinase from Sulfolobus Acidocaldarius by Matrix-Assisted Laser Desorption/ Ionization Mass Spectrometry. Analyst 2000, 125, 563-567.

22. Friess, S. D.; Daniel, J. M.; Zenobi, R. Probing the Surface Accessibility of Proteins with Noncovalent Receptors and MALDI Mass Spectrometry. Phys. Chem., Chem. Phys. 2004, 6, 2664-2675.

23. Farmer, T. B.; Caprioli, R. M. Assessing the Multimeric States of Proteins-Studies Using Laser Desorption Mass-Spectrometry. Biol. Mass Spectrom. 1991, 20, 796-800.

24. Bich, C.; Madler, S.; Chiesa, K.; DeGiacomo, F.; Bogliotti, N.; Zenobi, R. Reactivity and Applications of New Amino Reactive Cross-Linkers for Mass Spectrometric Detection of Protein-Protein Complexes. Anal. Chem. 2009, unpublished (submitted).

25. Cox, J. M.; Chan, C. A.; Chan, C.; Jourden, M. J.; Jorjorian, A. D.; Brym, M. J.; Snider, M. J.; Borders, C. L.; Edmiston, P. L. Generation of an Active Monomer of Rabbit Muscle Creatine Kinase by Site-Directed Mutagenesis: The Effect of Quaternary Structure on Catalysis and Stability. Biochemistry 2003, 42, 1863-1871.

26. Rao, J. K.; Bujacz, G.; Wlodawer, A. Crystal Structure of Rabbit Muscle Creatine Kinase. FEBS Lett. 1998, 439, 133-137.

27. Mannervik, B.; Jensson, H. Binary Combinations of Four Protein Subunits with Different Catalytic Specificities Explain the Relationship Between 6 Basic Glutathione S-Transferases in Rat-Liver Cytosol. J. Biol. Chem. 1982, 257, 9909-9912.

28. Hornby, J. A. T.; Codreanu, S. G.; Armstrong, R. N.; Dirr, H. W. Molecular Recognition at the Dimer Interface of a Class Mu Glutathione Transferase: Role of a Hydrophobic Interaction Motif in Dimer Stability and Protein Function. Biochemistry 2002, 41, 14238-14247.

29. Wallace, L. A.; Dirr, H. Folding and Assembly of Dimeric Human Glutathione Transferase A1-1. Biochemistry 1999, 38, 16686-16694.

30. Loo, R. R. O.; Goodlett, D. R.; Smith, R. D.; Loo, J. A. Observation of a Noncovalent Ribonuclease S-Protein S-Peptide Complex by Electrospray Ionization Mass-Spectrometry. J. Am. Chem. Soc. 1993, 115, 4391-4392.

31. Madler, S.; Bich, C.; Touboul, D.; Zenobi, R. Chemical Crosslinking with NHS Esters: A Systematic Study on Amino Acid Reactivities. J. Mass Spectrom. 2009, 44, 694-706.

32. Moitra, J.; Szilak, L.; Krylov, D.; Vinson, C. Leucine is the Most Stabilizing Aliphatic Amino Acid in the D Position of a Dimeric Leucine Zipper Coiled Coil. Biochemistry 1997, 36, 12567-12573.

33. Wendt, H.; Baici, A.; Bosshard, H. R. Mechanism of Assembly of a Leucine-Zipper Domain. J. Am. Chem. Soc. 1994, 116, 6973-6974.

34. Wendt, H.; Durr, E.; Thomas, R. M.; Przybylski, M.; Bosshard, H. R. Characterization of Leucine-Zipper Complexes by Electrospray-Ionization Mass-Spectrometry. Protein Sci. 1995, 4, 1563-1570. 